\title{
ABDOMINAL AORTIC ANEURYSM - COMPUTER MODELLING AND NUMERICAL SOLUTION
}

Original scientific paper

UDC: $519.61: 616.132$

https://doi.org/10.18485/aeletters.2021.6.2.5

\author{
S. Starcevic ${ }^{1,2 *}$, S. Savic ${ }^{1}$, N. Filipovic ${ }^{1,2}$, A. Milovanovic ${ }^{1}$, M. Djordjevic ${ }^{1}$ \\ ${ }^{1}$ Faculty of Engineering, University of Kragujevac, Serbia \\ ${ }^{2}$ BiolRC Research and Development Center of Bioengineering, Kragujevac, Serbia
}

\begin{abstract}
:
The main purpose of this study was to reconstruct 3D aorta models based on a series of 2D CT images of two patients suffering from an aneurysm. After the 3D models had been made, a numerical solution of the problem of the abdominal aortic aneurysm was obtained. The numerical solution incorporated mathematical models of biomechanical systems. Various parameters such as shear stress, pressure, and velocity of fluid through blood vessel could be calculated using these methods. The first part of the paper introduces the abdominal aorta and aneurysm. The second part of this paper describes the process of getting 3D model geometries of the aortic aneurysms. Finally, the results obtained from the models are discussed with the aim of predicting a rupture of an abdominal aortic aneurysm and selecting the right treatment for this disease.
\end{abstract}

\section{ARTICLE HISTORY}

Received: 17.05.2021.

Accepted: 20.06.2021.

Available: 30.06.2021.

\section{KEYWORDS}

Finite elements, 3D model, Aorta, Aneurysm, PAK-F solver

\section{INTRODUCTION}

The process of making models can be found in all spheres of life. The model has one purpose - to assess the influence of different parameters on the examined system. During the testing, the system is replaced by a model and thus the experiment is carried out. The accurateness of the results depends on the level of similarity between the model and the tested system - the greater the similarity the better the results.

Medical models are very important, and they have a wide application. The human body is an extremely complex system so it is impossible to make a perfect model that would unite all the systems that make up the human body. In practice, simplified models of individual organs or systems are used and combined.

This paper will show reconstructed geometries of two aortic aneurysms based on the data provided by CT images. The goal of this paper is to provide useful information that would help to select treatments for an abdominal aortic aneurysm.

\section{AORTA}

The aorta is a large blood vessel that starts from the heart, passes through the middle of the abdomen and splits into two arteries that supply blood to the legs. The aorta originates from the left ventricle of the heart, forms an arch and then extends to the abdomen where it branches into two smaller arteries which are called the renal arteries. The renal arteries have low resistance, so that two thirds of the input flow go to the abdominal aorta $[1,2]$.

\section{Abdominal aortic aneurysm}

An abdominal aortic aneurysm (AAA), shown in Figure 1 , is an enlargement lining the abdominal aorta, the largest blood vessel in the human body. An AAA is mostly caused by a localized damaged or weak spot of the blood vessel wall. Aneurysms are defined as arterial dilatations with a diameter $50 \%$ greater than the normal size. Therefore, an aorta with a diameter of $30 \mathrm{~mm}$ is defined as aneurysmal 
since the diameter of a normal aorta is about $20 \mathrm{~mm}$ [3-5].

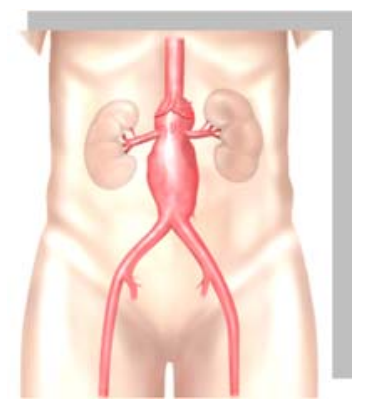

Fig. 1. Abdominal aortic aneurysm

\section{FLUID MECHANICS IN ARTERIES}

From the point of view of fluid mechanics, the cardiovascular system is considered as system in which the flow takes place and where viscous and inertial forces are dominant. The ratio between the viscous and inertial forces defines the Reynolds number whose values range from 1 in the small arteries to 4000 in the largest arteries [1]. The critical Reynolds number has a value of 2320 . Fluid motion is laminar if the value of the actual Reynolds number is less than the value of the critical Reynolds number, and the motion is turbulent if the value of the actual Reynolds number is greater than the value of the critical Reynolds number [6].

The Windkessel model is a simple onedimensional model which best explains the relations between the pressure and total blood flow (Figure 2.) [1]. This model presents a cardiovascular system as a system whose components are a capacitive part and a resistant part connected in a series. The aorta will act as a condenser that receives blood in the systole phase, while during the diastole the aorta releases the received blood through resistant channels to smaller arteries in other organs. The blood pressure and flow obtained with this model correspond to the values of these quantities measured in humans.

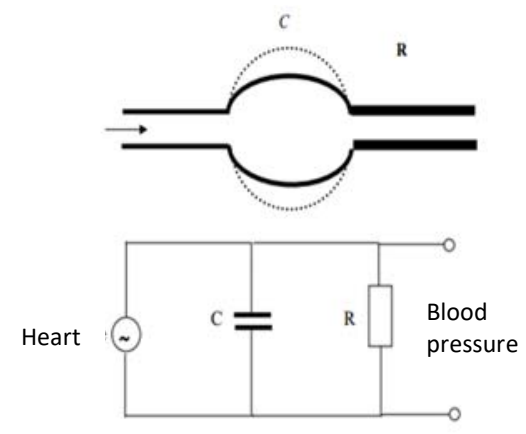

Fig. 2. One-dimensional model of the arterial system

\subsection{Continuity equation}

The law of mass conservation is one of the laws in fluids mechanics. The continuity equation is an analytical form of the law of mass conservation. The law of mass maintenance can be applied both to the elementary mass of a fluid particle $\mathrm{dm}$ and to the final mass $\Delta m$. According to this law, the mass of a fluid particle does not change during motion in a continuous current field:

$$
d m=\rho d V=\text { const }
$$

During the fluid motion, the density and the volume of particles can change, but their product must remain unchanged. In mathematical terms, this means that the total derivate of the elementary mass in time equals zero. The equation (1) can be written in a more concise form as:

$$
\frac{\partial \rho}{\partial t}+\operatorname{div}(\rho \vec{v})=0
$$

\subsection{Navier-Stokes equations}

The Navier-Stokes equations describe the motion of fluids. These equations arise from the application of Newton's Second Law to fluid motion, assuming that the stress in the fluid is the sum of the diffuse viscous term and the pressure term. These equations, together with the continuity equation, represent a "closed" system of equations and serve to determine the pressure $p$, the density $\rho$ and the fluid velocity $\vec{v}$. With the help of the formulated boundary conditions, they completely model the movement of fluids. The Navier-Stokes equations are nonlinear coupled partial differential equations of the second order. The continuity equation for the flow of an incompressible fluid, when $\rho=\rho_{0}=$ const, has a special form:

$$
\operatorname{div} \vec{v}=0,
$$

which yields the differential equation of the incompressible fluid motion, or the Navier-Stokes equation of the viscous liquid motion, which has the following vector form:

$$
\frac{d \vec{v}}{d t}=\vec{f}-\frac{1}{\rho} \operatorname{gradp}+v \Delta \vec{v} .
$$


The left side of the equation describes acceleration, while the right side is, in principle, the addition of forces. In the equation (4), $\vec{v}$ is the fluid velocity, $\rho$ is the fluid density, $p$ is the pressure, $f$ represents the forces acting on the fluid per unit volume, such as gravitational or centrifugal forces, and $v$ represents the kinematic viscosity and it is the quotient of dynamic viscosity $\mu$ and density of the fluid $(\mu / \rho)[6]$.

A significant feature of these equations is the presence of convective acceleration, which represents the effect of time-independent fluid acceleration with respect to space. While individual fluid particles experience time-dependent acceleration, the convective acceleration of the flow field is a spatial effect. The third term of the equation (4) represents convective acceleration, and it is a nonlinear quantity $[6,7-15]$.

\section{RECONSTRUCTED 3D GEOMETRIES OF AAA}

Reconstruction of patients' aorta geometry is the first step in simulation of the blood flow. The real geometries were reconstructed using Mimics, Geomagic and Femap software. Mimics was used for 2D image processing, while geometry was modified in Geomagic which was also used for modification of the finite element mesh. Femap was used to create and check finite element meshes. Some of previous studies had different methodology when it comes to reconstruction. One of previous papers also used CT images, but softwares used for reconstruction were different than one presented in this paper. Those softwares were Solid Edge V20, Solid Edge draft and Solid Edge Solid Part. Finite elements meshes were made using software Ansys Gambit 2.4. numerical analysis have been done in commercial softwer Ansys Fluent 13.0, where in this paper PAK- $F$ was used [16].

A set of images used for reconstruction was obtained from computed tomography. Every set required careful processing so it could give realistic AAA geometry. The main purpose of the 3D geometry reconstruction is making a realistic $3 \mathrm{D}$ lumen.

This study included two male patients with a diagnosed an aneurysm. For each patient a set of images contained about 750 images. The reconstruction steps will be shown in the following part of the paper.

Figures 3 and 4 present computed tomography (CT) images for the patients 1 and 2 , respectively.

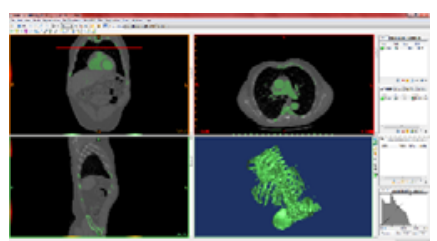

Fig. 3. CT images of the patient 1

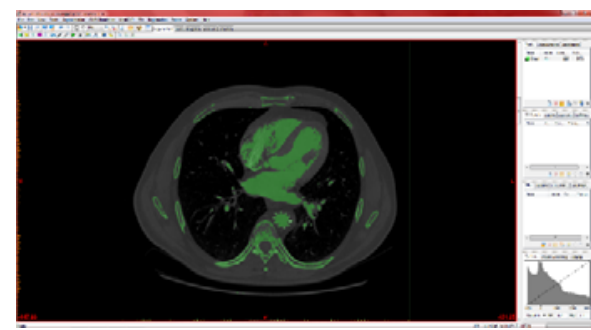

Fig. 4. CT image for the patient 2

Figures 5 and 6 show geometries of AAAs before and after the $3 D$ models were processed in the Geomagic software.
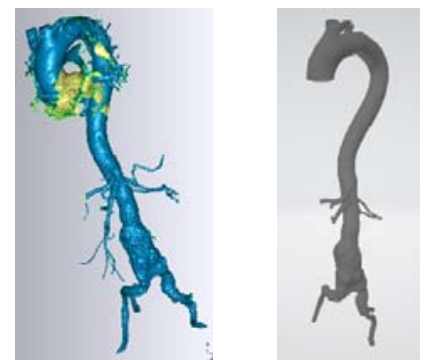

Fig. 5. 3D model of the patient's 1 AAA. Non-finished model (left), 3D geometry with good a finite element mesh
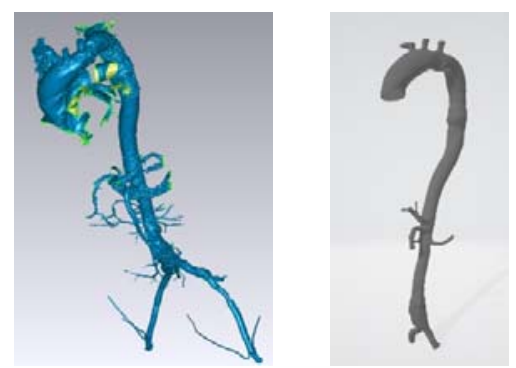

Fig. 6. 3D model of the patient's 2 AAA. Non-finished model (left), 3D geometry with a good finite element mesh

After creating the AAA 3D geometry, the next step was to create a finite element mesh. All the necessary modifications and preparations of the mesh were done in Geomagic. After the mesh had been generated, the inlet and outlet lying in the plane were made and the check for intersection between triangles done, the mesh was imported into Femap software where the mathematical model could be applied. PAK-F software was used for numerical solution of the AAA problems in this 
study. PAK-F solver is a package of solvers developed at the Faculty of Engineering in Kragujevac [17-19].

Figure 7 and 8 present 3D finished geometries ready to be used by PAK-F solver.

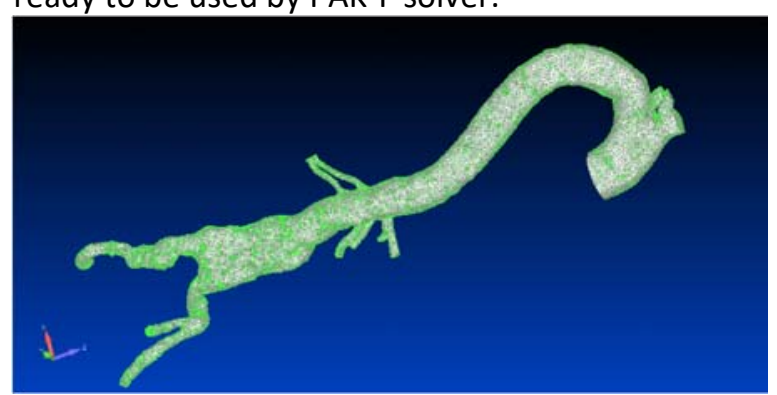

Fig. 7. Finished patient's 1 AAA geometry

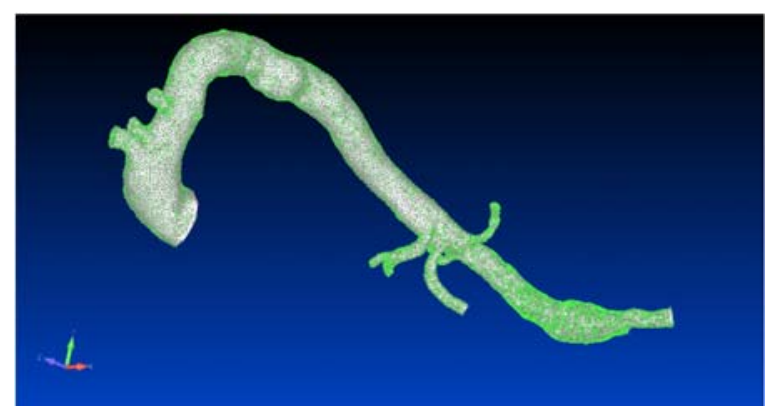

Fig. 8. Finished patient's 2 AAA geometry

\section{RESULTS AND DISCUSSION}

This paper presents results for two patients diagnosed with an aneurysm. This section of the paper presents numerical solutions.

The variables considered here: pressure, velocity, shear stress, wall stress cannot be measured non-invasively. However, based on computer simulations, very important information that can be obtained and used to predict the possibility of AAA rupture.

Blood is a non-Newtonian fluid. Its viscosity cannot be defined by a constant dynamic viscosity value because there is a nonlinear relationship between the shear rate and the shear stress [1].

The behaviour of blood must be taken into account when modelling. The initial conditions set at the entrance to the aorta are: blood density $\rho$ $=1.05 \mathrm{~g} / \mathrm{cm}^{3}$, kinematic viscosity of Newtonian fluid $v=0.035 \mathrm{~cm}^{2} / \mathrm{s}$. The input velocity is defined by the function of the input profile shown in the figure 9. This diagram represents the dependence of the flow on the relative time $t / T$. The input flow is assumed to be pulsating. The maximum is reached when the value $t / T=0.25$. The finite element network of the lumen of the first patient consists of 305629 nodes, and the lumen of the second patient has 313407 nodes.

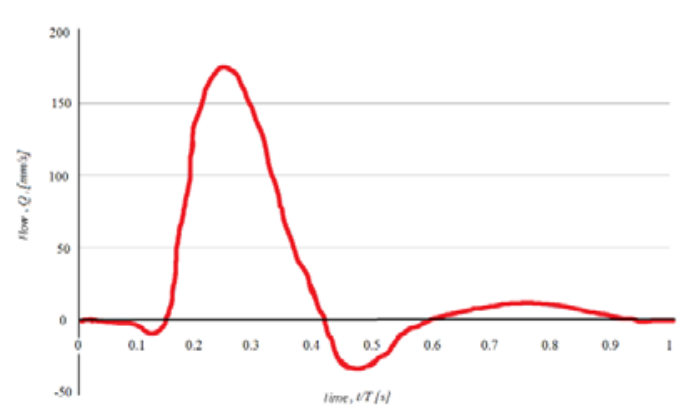

Fig. 9. A typical waveform at the entrance to the aorta

For the first patient, the solid consists of 135410 nodes in the finite element network, while the number of solid nodes for the second patient is 152741. The aorta with deformable walls is modelled according to the solid-fluid interaction algorithm. The blood data are the same as for the lumen modelling. The data used for the aortic wall are: Jung modulus of elasticity $E=2.7 \mathrm{MPa}$, Poisson's ratio $v=0.45 \mathrm{~cm}^{2} / \mathrm{s}$, tissue density $\rho$ $=1.1 \mathrm{~g} / \mathrm{cm}^{3}$ and wall thickness $\delta=0.2 \mathrm{~cm}$.

Figures 10 and 11 show the finite element meshes for the lumen and solid, respectively, made for the first patient.

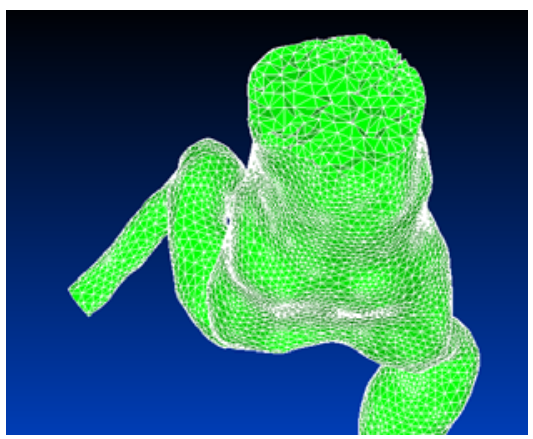

Fig. 10. Finite element mesh for the lumen of the patient 1

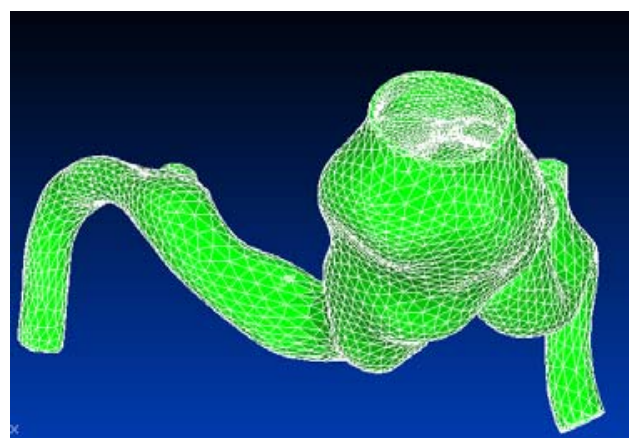

Fig. 11. Finite element mesh for the solid of the patient 1

Figure 12 shows blood velocity trajectories through the aorta. The trajectories are presented in different colours. For the first patient, the 
maximum blood velocity is $1850 \mathrm{~mm} / \mathrm{s}$, while the velocity of the blood velocity through the aorta of the second patient reaches a value of $1780 \mathrm{~mm} / \mathrm{s}$. The velocity values are high in the area of the ascending aorta and at the entrances to the coronary and subclavian arteries. The blood velocity in the ascending aorta has the highest values because blood is pumped directly from the heart in that area.

It can be noticed that the values of the velocities through the aneurysm for both patients, are very small, up to $400 \mathrm{~mm} / \mathrm{s}$. This is a consequence of a larger aortic diameter and the increase in volume due to the aneurysm. What happens in the area of an aneurysm is a classic example of fluid mechanics problem when fluid (in this case blood) encounters a sudden expansion. Then a local loss of flow energy occurs, and vortices are created, which turns a laminar flow into turbulent [6]. With the first patient, a vortex in the area of the aneurysm can be noticed, while with the second patient, a slight curvature of the velocity trajectory in the area of the aneurysm is seen. This vortex can be explained by a blood delay, and can serve as an indicator for an additional prognosis.
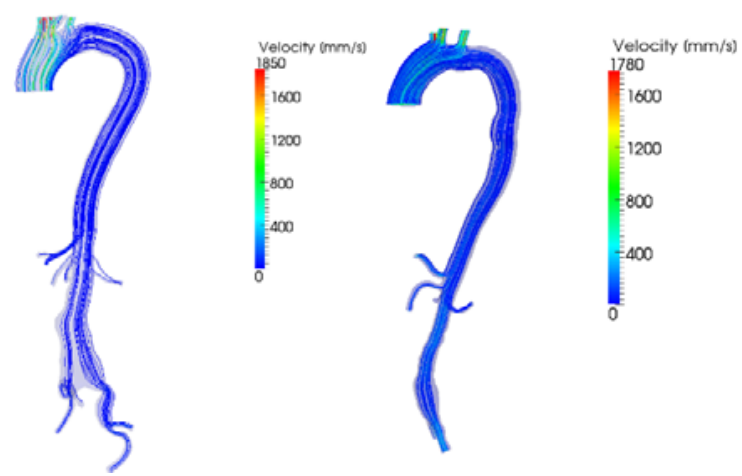

Fig. 12. The blood velocity trajectories of the patient 1 (left), and the patient 2 (right)

The shear stress was calculated, and the values are shown in Figures 13 and 14. These figures also show the pressure values in these aortas suffering from an aneurysm.

In both models, small values of the shear stress are read in the aneurysm zones. The greatest shear stresses are at the entrances of the coronary and subclavian arteries, as well as in the ascending aorta, which is expected, because it is a direct consequence of the fact that the ascending aorta starts directly from the heart. The pressure distribution is such that the pressure has very high values in the area of the ascending aorta, in both patients, which is also a direct consequence of the fact that the blood from the heart is pumped into it.
The pressure in the aorta of the first patient in the area of the aneurysm is very high. This pressure is significantly higher compared to the value of the pressure in the aneurysm of the second patient. This could have been expected since the first patient's aneurysm is much larger in size than the second patient's aneurysm. If the maximum values of the pressure and the shear stress are compared, it can be seen that the maximum pressure of the first patient is lower than the maximum pressure of the second patient, while the opposite is the case for the maximum values of the shear stresses.
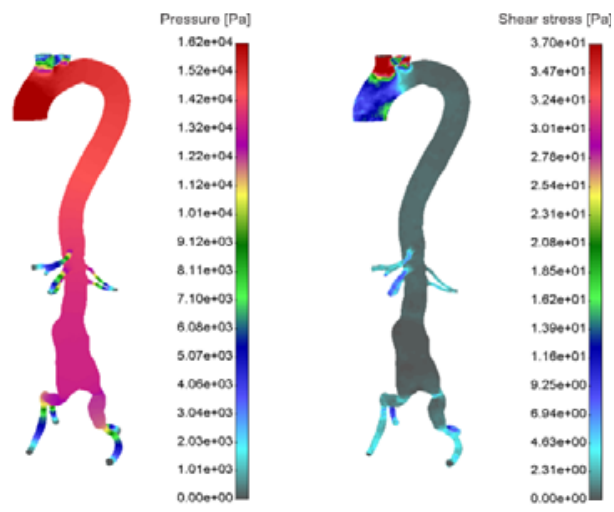

Fig. 13. Total pressure and shear stress for the patient 1
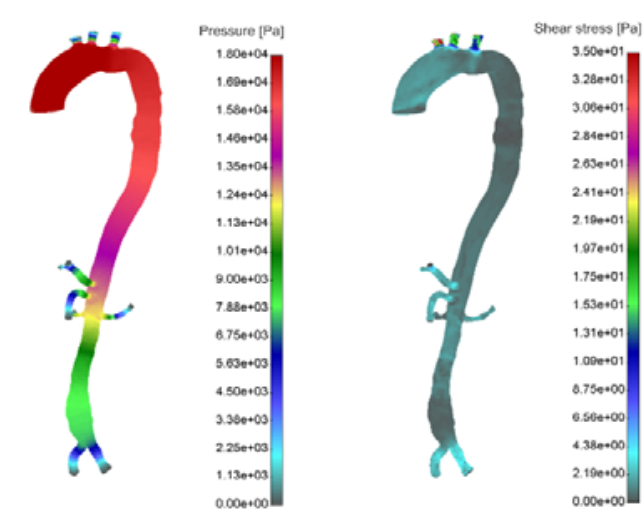

Fig. 14. Total pressure and shear stress for the patient 2

After these calculations, the aortic wall tension was determined in order to predict the rupture of the abdominal aortic aneurysm. The results are shown in Figures 15 and 16. Based on the aortic wall tension, the site of rupture can be predicted.

Figure 15 shows that the entire surface of the aneurysm is dominated by the pressure values marked blue on the scale. Slightly higher tensions are noticed, with values around $1.38 \mathrm{e}+05 \mathrm{~Pa}$. In the aneurysm of the first patient, pressure values range from $2.50 \mathrm{e}+04 \mathrm{~Pa}$ up to the maximum value of $2.00 \mathrm{e}+05 \mathrm{~Pa}$, which is observed as a red dot on the wall. Furthermore, this area with the maximum stress presents the most critical area in which an 
aneurysm rupture can occur. Of course, a rupture can also occur in the stress range between $1.25 \mathrm{e}+05$ - 1.38e+05 Pa. These areas are marked yellow.

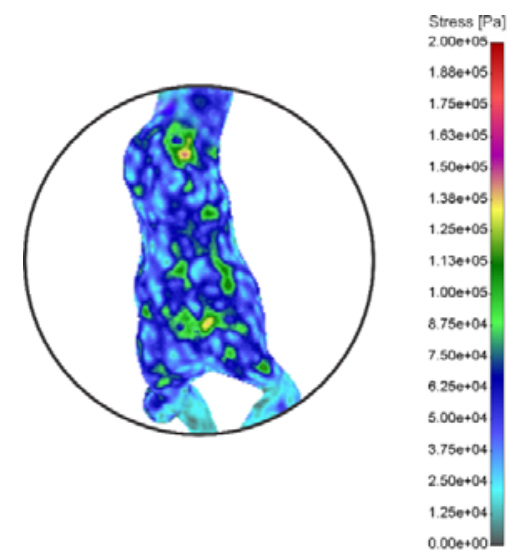

Fig. 15. Distribution of tension $(\mathrm{Pa})$ for the patient 1

Figure 16 shows that the maximum stress on the aortic wall of the second patient is $1.70 \mathrm{e}+05 \mathrm{~Pa}$, which is also lower than the maximum stress in the aortic wall of the first patient. The aneurysmal wall is characterised by the highest stress ranging from 7.44e+04 $\mathrm{Pa}$ to 1.17e+05 $\mathrm{Pa}$. A rupture may occur in the area marked yellow. The problem is more serious in the case of the patient 1.

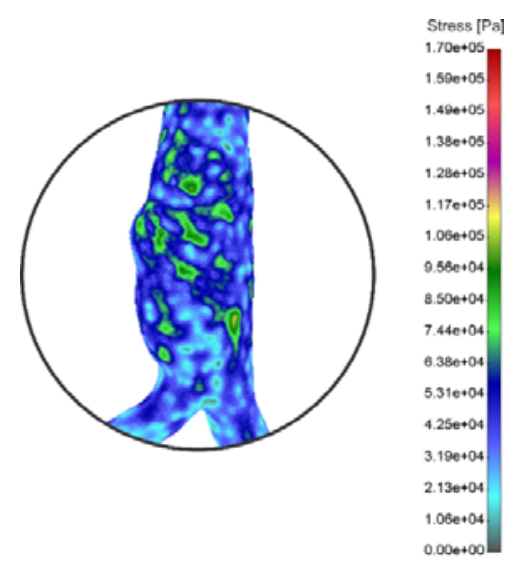

Fig. 16. Distribution of tension $(\mathrm{Pa})$ for the patient 2

Based on the presented results, it can be concluded that, despite the visible difference in aneurysm diameters, the values of shear stress, pressure, and stress in the aortic wall are of the same order of magnitude. It can also be concluded that the first patient is in a very critical condition, because the possibility of the aneurysm rupture is very high. However, as the strength of the wall varies for each patient, the wall stress is not a $100 \%$ accurate criterion, but it is very useful to doctors while choosing a treatment for the disease.

In some previous studies, researchers had different approaches. Some of studies which are different from the one presented in this paper included comparison between two cases. One of those cases included blood flow analysis in aortic system with abdominal aortic aneurism and left common iliac aneurysm before stent graft placement, while in second case was included stent graft geometry, at the site of the aneurysm. This analysis were performed also using the possibilities of computational fluid dynamics. For those two cases, the velocity, pressure and shear stress were visualized and compared [9].

\section{CONCLUSION}

This paper presents 3D models of abdominal aortic aneurysms, the process of their modelling and the results of simulations made using these models. In order for the equation system to be solved, some approximations need to be made. Theoretically, it is possible to make a model that will perfectly describe an AAA, but then a special model must be made for each patient, which would be a problem. The equations needed to obtain the model would be very complex and impossible to solve. Therefore, approximations are necessary. The type of approximations used depends on the accuracy of the model and its purpose.

The models presented in this paper have application in clinical practice. Using the appropriate software packages, it is possible to predict how the disease will develop and what will happen to the observed aorta. Based on the calculated parameters, the probability of rupture can be calculated, and treatment options can be analysed for a particular patient.

These software packages are coming into clinical practice, but they have not reached widespread use yet. The benefits of these computer methods to medicine are apparent and so is the need for their improvement. In future, the improved methods could be used to identify high-risk aneurysms, to determine the risk for each patient individually, to detail the geometric description of AAAs and to closely monitor the growth of the aneurysm.

Despite its limitations, rapid technological development provides mathematical modelling with the potential to become one of the main aids 
in determining the risk for sick people and in making decisions about therapies. All these advantages make such methods suitable for the $21^{\text {st }}$ century.

\section{REFERENCES}

[1] N. Filipovic, Basics of Bioengineering. University of Kragujevac, Faculty of Engineering, Kragujevac, 2012.

[2] M. Kojic, N. Filipovic, I. Vlastelica, M. Zivkovic, Modeling of blood flow in the human aorta with use of an orthotropic nonlinear material model for the walls. Proceedings Second MIT Conference on Compurational Fluid and Solid Mechanics, Boston, USA, 17-20 June 2003, 1751-1754.

https://doi.org/10.1016/B978-008044046-

0.50427-9

[3] T. Fukushima, T. Matsuzawa, T. Homma, Visualization and finite element analysis of pulsatile flow in models of the abdominal aortic aneurysm. Biorheology, 26 (2), 1989: 109-130.

https://doi.org/10.3233/BIR-1989-26203

[4] V. Jokovic, Abdominal aortic aneurysm. University of Kragujevac, Faculty of Medical Sciences, Kragujevac, 2012.

[5] D.A. Vorp, M.L. Raghavan, M.W. Webster, Mechanical wall stress in abdominal aortic aneurysm: Influence of diameter and asymmetry. Journal of Vascular Surgery, 27 (4), 1998: $632-639$.

https://doi.org/10.1016/S0741-5214(98)70227-7

[6] B. Obrovic, Fluid mechanics. University of Kragujevac, Faculty of Mechanical Engineering, Kragujevac, 2007.

[7] V. Paramasivam, N. Filipovic, K. Muthusamy, M.R.A. Kadir, Finite Element Computation for Solving Pulsatile Blood Flow: Relevance in Assessing the Flow Dynamics in Abdominal Aortic Aneurysms. CFD letters, 2 (4), 2010: 149162.

[8] V. Isailovic, I. Koncar, D. Veljkovic, D. Mikasinovic, D. Nikolic, M. Markovic, L. Davidovic, M. Kojic, N. Filipovic, Computer model and clinical relevance of abdominal aorta aneurysm with compliant nonlinear material wall. Proceedings of the $10^{\text {th }}$ IEEE International Conference on Information Technology and Applications in Biomedicine, 35 November, Corfu, Greece, 2010, pp.1-4. https://doi.org/10.1109/ITAB.2010.5687681
[9] S. Djorovic, I. Koncar, L. Davidovic, S. Starcevic, N. Filipovic, Computational Analysis of Blood Flow Characteristics in an Aortic System with Abdominal and Left Common Iliac Aneurysm Pre- and Post-Stent Grafting. EAl Endorsed Transaction on Pervasive Health and Tecnology, 13 (4), 2018: e4.

http://dx.doi.org/10.4108/eai.28-2-2018.154145

[10] A.A. Noel, P. Gloviczki, K.J. Cherry Jr, T.C. Bower, J.M. Panneton, G.I. Mozes, W.S. Harmsen, G.D. Jenkins, J.W. Hallett Jr: Ruptured Abdominal Aortic Aneurysm: The Excessive Mortality of Conventional Repair. Journal of Vascular Surgery, 34 (1), 2001: 41 - 46.

https://doi.org/10.1067/mva.2001.115604

[11] V. Paramasivam, N. Filipovic, K. Muthusamy, M. R. A. Kadir, Finite Element Modeling for Solving Pulsatile Flow in a Fusiform Abdominal Aortic Aneurysm. Biomedicine international, 1 (2), 2010: 52-61.

[12] P.M. Brown, D.T. Zelt, B. Sobolev, The Risk of Rupture in Untreated Aneurysms: The Impact of Size, Gender, and Expansion Rate. J. Vasc. Surg., 37 (2), 2003: 280-284.

https://doi.org/10.1067/mva.2003.119

[13] F. Gao, M. Watanabe, T. Matsuzawa, Stress Analysis in a Layered Aortic Arch Model Under Pulsatile Blood Flow. BioMed. Eng. Online, 5 2006: 25.

https://doi.org/10.1186/1475-925X-5-25

[14] Z. Sun, T. Chaichana, M. Sangworasil, S. Tungjitkusolmun, Computational Fluid Analysis of Blood Flow Characteristics in Abdominal Aortic Aneurysms Treated with Suprarenal Endovascular Grafts. In: Lim C.T., Goh J.C.H. (eds). 13 ${ }^{\text {th }}$ International Conference on Biomedical Engineering. IFMBE Proceedings, Springer, Berlin, Heidelberg. 2009, Vol.23, 1728-1732

https://doi.org/10.1007/978-3-540-92841-6 429

[15] B.J. Wolters, M.C. Rutten, G.W. Schurink, U. Kose, J. de Hart, F.N. van de Vosse, A PatientSpecific Computational Model of FluidStructure Interaction in Abdominal Aortic Aneurysms. Med. Eng. Phys., 27 (10), 2005: 871-883.

https://doi.org/10.1016/i.medengphy.2005.06.008

[16] A. Toth, Abdominal aortic aneurysm modelling using computed fluid dynamics, University of Novi Sad, Faculty of Science and Mathematics, Novi Sad, 2013. 
[17] M. Kojic, N. Filipovic, M. Zivkovic, R. Slavkovic, N. Grujovic, PAK-F, Program for FE Analysis of Fluid Flow with Heat Transfer, Users Manual. Faculty of Mechanical Engineering of Kragujevac-Laboratory for Engineering Software, Kragujevac, Serbia, 1999.

[18] M. Kojic, N. Filipovic, B. Stojanovic, N. Kojic, Computer modelling in bioengineering: theoretical background, examples and software, Chichester, England: Wiley, 2008.

[19] M. Kojic, N. Filipovic, M. Zivkovic, R. Slavkovic, N. Grujovic, PAK-F finite element program for laminar flow of incompressible fluid and heat transfer. Laboratory for engineering software, Faculty of mechanical engineering, University of Kragujevac, Kragujevac, Serbia-Yugoslavia, 1998. 